\title{
DEGUM und DRG schließen Kooperationsvereinbarung
}

Die „Arbeitsgemeinschaft Ultraschall“ in der Deutschen Röntgengesellschaft (DRG) und die „Sektion Radiologie“ der Deutschen Gesellschaft für Ultraschall in der Medizin (DEGUM) werden in Zukunft stärker zusammenarbeiten. Das haben beide Seiten in einer Kooperationsvereinbarung beschlossen.

Die Zusammenarbeit soll vor allem in den Bereichen Fort- und Weiterbildung, Wissenschaft und Forschung stattfinden. Ziel ist es, die gegenseitige Unterstützung durch verschiedene Projekte umzusetzen. Zu diesen Projekten zählen mehr Kooperation bei der Leitlinienarbeit und eine stärkere Abstimmung von Zertifizierungen in den Bereichen Sonografie und des DEGUM-Stufenkonzepts.

Zusätzlich wollen sich die „AG Ultraschall“ in der DRG und die „Sektion Radiologie“ der DEGUM regelmäßig, unter anderem auf den jeweiligen Mitgliederversammlungen, fachlich austauschen, kooptierte Vertreter/Vertreterinnen in die jeweiligen Leitungsgremien entsenden und stärker bei der Programmplanung und -gestaltung von Jahrestagungen und Fortbildungsveranstaltungen kooperieren.

Die Vorstände der beiden Gesellschaften begrüßen die Zusammenarbeit. 